Pacific

Journal of

Mathematics

ISOMORPHISMS OF TYPE $A$ AFFINE HECKE ALGEBRAS AND MULTIVARIABLE ORTHOGONAL POLYNOMIALS

T.H. BAKer AND P.J. Forrester

Volume $194 \quad$ No. 1

May 2000 


\title{
ISOMORPHISMS OF TYPE $A$ AFFINE HECKE ALGEBRAS AND MULTIVARIABLE ORTHOGONAL POLYNOMIALS
}

\author{
T.H. BAKER AND P.J. ForRester
}

\begin{abstract}
We examine two isomorphisms between affine Hecke algebras of type $A$ associated with parameters $q^{-1}, t^{-1}$ and $q, t$. One of them maps the non-symmetric Macdonald polynomials $E_{\eta}\left(x ; q^{-1}, t^{-1}\right)$ onto $E_{\eta}(x ; q, t)$, while the other maps them onto non-symmetric analogues of the multivariable Al-Salam \& Carlitz polynomials. Using the properties of $E_{\eta}\left(x ; q^{-1}, t^{-1}\right)$, the corresponding properties of these latter polynomials can then be elucidated.
\end{abstract}

\section{Introduction.}

In several recent works [28]-[29], [9]-[10], eigenstates of the rational (type $A$ ) Calogero-Sutherland model have been investigated from an algebraic point of view. In particular it has been shown that the algebra governing the eigenfunctions of the periodic Calogero-Sutherland model (namely the type $A$ degenerate affine Hecke algebra augmented by type $A$ Dunkl operators) is isomorphic to its rational model counterpart. This enables information to be gleaned about the properties of the eigenfunctions in the rational case (the (non-)symmetric Hermite polynomials) from the corresponding periodic eigenfunctions (the (non-)symmetric Jack polynomials).

To summarize the argument, consider the type $A$ Dunkl operators

$$
d_{i}:=\frac{\partial}{\partial x_{i}}+\frac{1}{\alpha} \sum_{p \neq i} \frac{1-s_{i p}}{x_{i}-x_{p}}
$$

which, along with the operators representing multiplication by the variable $x_{i}$ and the elementary transpositions $s_{i j}$, satisfy the following commutation relations

$$
\begin{aligned}
& {\left[d_{i}, x_{j}\right]=\left\{\begin{array}{cc}
-\frac{1}{\alpha} s_{i j} & i \neq j \\
1+\frac{1}{\alpha} \sum_{p \neq i} s_{i p} & i=j
\end{array}\right.} \\
& d_{i} s_{i p}=s_{i p} d_{p} \quad\left[d_{i}, s_{j p}\right]=0, \quad i \neq j, p .
\end{aligned}
$$

It is easily checked that the map $\rho$ defined by

$$
\rho\left(x_{i}\right)=x_{i}-\frac{1}{2} d_{i}, \quad \rho\left(d_{i}\right)=d_{i}, \quad \rho\left(s_{i j}\right)=s_{i j}
$$


is an isomorphism of the algebra (1.1) [28].

Now, the non-symmetric Jack polynomials $E_{\eta}(x)$, indexed by compositions $\eta:=\left(\eta_{1}, \ldots, \eta_{n}\right)$ can be defined [23] as the unique eigenfunctions of the mutually commuting Cherednik operators

$$
\xi_{i}:=\alpha x_{i} d_{i}+\sum_{p>i} s_{i p}-n+1
$$

with a unique expansion of the form

$$
E_{\eta}(x)=x^{\eta}+\sum_{\nu<\eta} c_{\eta \nu} x^{\nu}
$$

Here, the partial order $<$ is defined on compositions by: $\nu<\eta$ iff $\nu^{+}<$ $\eta^{+}$with respect to the dominance order (where $\nu^{+}$is the unique partition associated to $\nu$ etc) or $\nu^{+}=\eta^{+}, \nu \neq \eta$ and $\sum_{i=1}^{p}\left(\eta_{i}-\nu_{i}\right) \geq 0$, for all $p=1, \ldots, n$. The polynomial $E_{\eta}(x)$ is an eigenfunction of $\xi_{i}$ given by (1.3) with eigenvalue

$$
\bar{\eta}_{i}=\alpha \eta_{i}-\#\left\{k<i \mid \eta_{k} \geq \eta_{i}\right\}-\#\left\{k>i \mid \eta_{k}>\eta_{i}\right\} .
$$

Using the isomorphism (1.2) it follows that the polynomials $[\mathbf{2 7}, \mathbf{2 4}, \mathbf{1 0}]$

$$
E_{\eta}^{(H)}(x):=E_{\eta}(\rho(x)) \cdot 1
$$

are eigenfunctions of the operators

$$
h_{i}=\rho\left(\xi_{i}\right)=\xi_{i}-\frac{\alpha}{2} d_{i}^{2}
$$

which are precisely the eigenoperators of the non-symmetric Hermite polynomials [2]. The orthogonality of these latter polynomials with respect to the usual multivariable Hermite inner product then follows from the fact that the operator (1.6) is self-adjoint with respect to the inner product

$$
\langle f, g\rangle:=\prod_{i=1}^{n} \int_{-\infty}^{\infty} d x_{i} e^{-x_{i}^{2}} \prod_{1 \leq j<k \leq n}\left|x_{j}-x_{k}\right|^{2 / \alpha} f(x) g(x) .
$$

In this work, we provide a similar analysis of the Macdonald case. As such, we introduce an isomorphism of the $q$-analogue of the algebra (1.1), namely the subalgebra $\mathcal{S}_{q, t}:=\left\{T_{i}, \omega, D_{i}, x_{i}\right\}$ of the algebra of endomorphisms of the polynomial ring $\mathbb{Q}(q, t)\left[x_{1}, \ldots, x_{n}\right]$. Here, $\left\{T_{i}, \omega\right\}$ generate a subalgebra isomorphic to the (type $A$ ) affine Hecke algebra, while $\left\{D_{i}\right\}$ are the $q$-Dunkl operators introduced in $[3, \mathbf{3}]$. To describe this mapping, we need to introduce some further concepts.

The generalization of the formalism of non-symmetric Jack polynomials to the Macdonald case involves replacing the Cherednik operators (1.3) by their $q$-analogues which can be realized as a commutative subalgebra of the 
affine Hecke algebra [18]. In the type $A$ case, one can describe this using the Demazure-Lustig operators

$$
\begin{array}{rlrl}
T_{i} & :=t+\frac{t x_{i}-x_{i+1}}{x_{i}-x_{i+1}}\left(s_{i}-1\right) & & \\
T_{0} & :=t+\frac{q t x_{n}-x_{1}}{q x_{n}-x_{1}}\left(s_{0}-1\right) &
\end{array}
$$

along with the operator

$$
\omega:=s_{n-1} \cdots s_{2} s_{1} \tau_{1}=s_{n-1} \cdots s_{i} \tau_{i} s_{i-1} \cdots s_{1} .
$$

Here $\tau_{i}$ is the operator which replaces $x_{i}$ by $q x_{i}, s_{i}:=s_{i, i+1}$ for $1 \leq i \leq n-1$ and $s_{0}:=\omega s_{1} \omega^{-1}$. The affine Hecke algebra is then generated by elements $T_{i}, 0 \leq i \leq n-1$ and $\omega$, satisfying the relations

$$
\begin{aligned}
\left(T_{i}-t\right)\left(T_{i}+1\right) & =0 \\
T_{i} T_{i+1} T_{i} & =T_{i+1} T_{i} T_{i+1} \\
T_{i} T_{j} & =T_{j} T_{i} \quad|i-j| \geq 2 \\
\omega T_{i} & =T_{i-1} \omega .
\end{aligned}
$$

There is a commutative subalgebra generated by elements of the form $[\mathbf{5}, \mathbf{6}]$

$$
Y_{i}:=t^{-n+i} T_{i} \cdots T_{n-1} \omega T_{1}^{-1} \cdots T_{i-1}^{-1}
$$

which have the following relations with the generators $T_{i}$ for $1 \leq i \leq n-1$.

$$
T_{i} Y_{i+1}=t Y_{i} T_{i}^{-1}, \quad T_{i} Y_{i}=Y_{i+1} T_{i}+(t-1) Y_{i}, \quad\left[T_{i}, Y_{j}\right]=0, \quad j \neq i, i+1 .
$$

The non-symmetric Macdonald polynomials $E_{\eta}(x ; q, t)$ are defined as the simultaneous eigenfunctions of the commuting operators $Y_{i}$ with an expansion of the form (1.4). The corresponding eigenvalue is $t^{\bar{\eta}_{i}}$ with $\bar{\eta}_{i}$ given in (1.5), and $t^{\alpha}=q$. From now on, we drop the dependence on $q$ and $t$ and just write $E_{\eta}(x) \equiv E_{\eta}(x ; q, t)$ when the meaning is unambiguous.

Define the following degree-raising operator

$$
e_{i}:=t^{i-1} T_{i} \cdots T_{n-1} x_{n} \omega T_{1}^{-1} \cdots T_{i-1}^{-1} .
$$

Using (1.12)-(1.14) it can be shown that the operators $e_{i}$ form a set of mutually commuting operators. Our first result is:

Theorem 1.1. We have

$$
E_{\eta}\left(e_{1}, \ldots, e_{n} ; q^{-1}, t^{-1}\right) .1=\alpha_{\eta}(q, t) E_{\eta}\left(x_{1}, \ldots, x_{n} ; q, t\right)
$$

where

$$
\alpha_{\eta}(q, t)=q^{\sum_{i}\left(\begin{array}{c}
\eta_{i} \\
2
\end{array}\right)} t^{\sum_{i}(n-i) \eta_{i}^{+}-\ell\left(w_{\eta}\right)}
$$

with $\ell\left(w_{\eta}\right)$ the length of the (unique) minimal permutation sending $\eta$ to $\eta^{+}$. 
The symmetric Al-Salam \& Carlitz (ASC) polynomials were examined in [1] as $q$-analogues of multivariable Hermite polynomials. There are two families of ASC polynomials, denoted $U_{\lambda}^{(a)}(x ; q, t)$ and $V_{\lambda}^{(a)}(x ; q, t)$, which are simply related by

$$
V_{\lambda}^{(a)}\left(x ; q^{-1}, t^{-1}\right)=U_{\lambda}^{(a)}(x ; q, t) .
$$

The polynomials $V_{\lambda}^{(a)}$ can be defined as the unique polynomials of the form

$$
V_{\lambda}^{(a)}(x ; q, t)=P_{\lambda}(x ; q, t)+\sum_{\mu<\lambda} b_{\lambda \mu} P_{\mu}(x ; q, t)
$$

which are orthogonal with respect to the inner product

$$
\begin{aligned}
\langle f, g\rangle^{(V)} & :=\int_{[1, \infty]^{n}} f(x) g(x) d_{q} \mu^{(V)}(x), \\
d_{q} \mu^{(V)}(x) & :=\Delta_{q}^{(k)}(x) \prod_{l=1}^{n} w_{V}\left(x_{l} ; q\right) d_{q} x_{l} .
\end{aligned}
$$

Here, $P_{\lambda}(x ; q, t)$ denotes the symmetric Macdonald polynomial [19] and we use the notation for $q$-integrals

$$
\int_{1}^{\infty} f(x) d_{q} x:=(1-q) \sum_{n=0}^{\infty} f\left(q^{-n}\right) q^{-n}
$$

while

$$
\begin{aligned}
w_{V}(x ; q) & =\frac{(q ; q)_{\infty}\left(\frac{1}{a} ; q\right)_{\infty}(q a ; q)_{\infty}}{(x ; q)^{\prime}{ }_{\infty}\left(\frac{x}{a} ; q\right)_{\infty}} \\
\Delta_{q}^{(k)}\left(x_{1}, \ldots, x_{n}\right) & :=\prod_{p=-(k-1)}^{k} \prod_{1 \leq i<j \leq n}\left(x_{i}-q^{p} x_{j}\right),
\end{aligned}
$$

where the dash in $(x ; q)_{\infty}^{\prime}$ denotes that any vanishing factor is to be deleted, and it is assumed $a<0$. Moreover, in (1.20) and in what follows, we assume $t=q^{k}$, where $k$ is a positive integer.

The polynomials $U_{\lambda}^{(a)}$ are orthogonal with respect to the inner product

$$
\begin{aligned}
\langle f \mid g\rangle^{(U)} & :=\int_{[a, 1]^{n}} f(x) g(x) d_{q} \mu^{(U)}(x), \\
d_{q} \mu^{(U)}(x) & :=\Delta_{q}^{(k)}(x) \prod_{l=1}^{n} w_{U}\left(x_{l} ; q\right) d_{q} x_{l}
\end{aligned}
$$

where $\Delta_{q}^{(k)}$ is given by (1.22) and

$$
w_{U}^{(a)}(x ; q):=\frac{(q x ; q)_{\infty}\left(\frac{q x}{a} ; q\right)_{\infty}}{(q ; q)_{\infty}(a ; q)_{\infty}\left(\frac{q}{a} ; q\right)_{\infty}}
$$


(1.25) $\int_{a}^{1} f(x) d_{q} x:=(1-q)\left(\sum_{n=0}^{\infty} f\left(q^{n}\right) q^{n}-a \sum_{n=0}^{\infty} f\left(a q^{n}\right) q^{n}\right), \quad(a<0)$.

This can be regarded as a consequence of (1.19), and the formulas

$$
\begin{gathered}
\left.\frac{1}{1-q} \int_{a}^{1} w_{U}^{(a)}(x ; q) f(x) d_{q} x\right|_{q \mapsto q^{-1}}=\frac{1}{1-q} \int_{1}^{\infty} w_{V}^{(a)}(x ; q) f(x) d_{q} x \\
\Delta_{q^{-1}}^{(k)}(x)=q^{-k n(n-1)} \Delta_{q}^{(k)}\left(x^{R}\right)
\end{gathered}
$$

where $x^{R}=\left(x_{n}, x_{n-1}, \ldots, x_{1}\right)$. The formula $(1.26)$ is established in
[1, eq. (2.23)], while (1.27) follows immediately from the definition (1.22).

Non-symmetric analogues of the ASC polynomials can be introduced in the following manner: Consider the following $q$-analogues of the type $A$ Dunkl operators $[\mathbf{8}]$ examined in $[\mathbf{3}]$,

$$
D_{i}:=x_{i}^{-1}\left(1-t^{n-1} T_{i}^{-1} \cdots T_{n-1}^{-1} \omega T_{1}^{-1} \cdots T_{i-1}^{-1}\right)
$$

and let

$$
E_{i}:=D_{i}+\left(1+a^{-1}\right) t^{n-1} Y_{i}-a^{-1} e_{i} .
$$

The operators $E_{i}$ mutually commute, and our second main result is that:

Theorem 1.2. The polynomials

$$
E_{\eta}^{(V)}(x ; q, t)=\frac{(-a)^{|\eta|}}{\alpha_{\eta}(q, t)} E_{\eta}\left(E ; q^{-1}, t^{-1}\right) \cdot 1
$$

where $\alpha_{\eta}(q, t)$ is given by (1.18) are the unique polynomials with an expansion of the form

$$
E_{\eta}^{(V)}(x ; q, t)=E_{\eta}(x ; q, t)+\sum_{|\nu|<|\eta|} c_{\eta \nu} E_{\nu}(x ; q, t)
$$

which are orthogonal with respect to the inner product (1.20). Furthermore, these polynomials are simultaneous eigenfunctions of the commuting family of eigenoperators

$$
h_{i}=Y_{i}+(1+a) t^{1-n} D_{i}+a t^{2-2 n} D_{i} Y_{i}^{-1} D_{i}
$$

with eigenvalue $t^{\bar{\eta}_{i}}$.

An immediate consequence of Thm. 1.2, (1.19), and (1.26), (1.27) is:

Corollary 1.3. The polynomials

$$
E_{\eta}^{(U)}(x ; q, t):=E_{\eta}^{(V)}\left(x^{R} ; q^{-1}, t^{-1}\right)
$$

are the unique polynomials with an expansion of the form

$$
E_{\eta}^{(U)}(x ; q, t)=E_{\eta}\left(x^{R} ; q^{-1}, t^{-1}\right)+\sum_{|\nu|<|\eta|} d_{\eta \nu} E_{\nu}\left(x^{R} ; q^{-1}, t^{-1}\right)
$$


which are orthogonal with respect to the inner product (1.23). These polynomials are simultaneous eigenfunctions of the operators $\hat{h}_{i}$, where $\hat{h}_{i}$ denotes the operator (1.31) modified by the involution ", which is defined by the mappings $q \mapsto q^{-1}, t \mapsto t^{-1}$ and $x_{i} \mapsto x_{n+1-i}$.

In Section 2, we examine the various properties of non-symmetric Macdonald polynomials used in subsequent calculations, including raising and lowering operators, and introduce a non-symmetric analogue of Kaneko's kernel [11]. We finish the section with a proof of Thm. 1.1. An isomorphism between Hecke algebras is introduced in Section 3, facilitating a proof of Thm. 1.2. Various properties of these non-symmetric ASC polynomials are then described including their normalization and a generating function. We conclude by clarifying their relationship to the non-symmetric analogues of the shifted Macdonald polynomials.

\section{Non-symmetric Macdonald polynomials.}

In this section we gather together some (old and new) results concerning non-symmetric Macdonald polynomials $E_{\eta}(x)$ in preparation of the proof of Thm. 1.1, as well as the forthcoming section on the non-symmetric ASC polynomials.

For future reference we note that the operators $T_{i}$ and $\omega$ defined by (1.8) and (1.10) have the properties

$$
\begin{array}{rc}
T_{i}^{-1} x_{i+1}=t^{-1} x_{i} T_{i} & T_{i}^{-1} x_{i}=x_{i+1} T_{i}^{-1}+\left(t^{-1}-1\right) x_{i} \\
(2.1) T_{i} x_{i}=t x_{i+1} T_{i}^{-1} & T_{i} x_{i+1}=x_{i} T_{i}+(t-1) x_{i+1} \\
\omega x_{1}=q x_{n} \omega & \omega x_{i+1}=x_{i} \omega
\end{array}
$$

valid for $1 \leq i \leq n-1$. Also note the following action of $T_{i}$ on monomials

$$
T_{i} x_{i}^{a} x_{i+1}^{b}= \begin{cases}(1-t) x_{i}^{a-1} x_{i+1}^{b+1}+\cdots+(1-t) x_{i}^{b+1} x_{i+1}^{a-1}+x_{i}^{b} x_{i+1}^{a} & a>b \\ t x_{i}^{a} x_{i+1}^{a} & a=b \\ (t-1) x_{i}^{a} x_{i+1}^{b}+\cdots+(t-1) x_{i}^{b-1} x_{i+1}^{a+1}+t x_{i}^{b} x_{i+1}^{a} & a<b .\end{cases}
$$

There exists a variant of the $q$-Dunkl operator (1.28) which is relevant to the forthcoming discussion. With ^ denoting the involution defined in the statement of Corollary 1.3, this operator is defined as

$$
\begin{aligned}
\mathcal{D}_{i} & :=-q \hat{D}_{n+1-i} \\
& =-q x_{i}^{-1}\left(1-t^{-n+1} T_{i-1} \cdots T_{1} \omega^{-1} T_{n-1} \cdots T_{i}\right) \\
& =q t^{-2 n+i+1} D_{i} Y_{i}^{-1} T_{i} \cdots T_{n-1} T_{n-1} \cdots T_{i} .
\end{aligned}
$$

In obtaining the first equality in (2.3), the facts that

$$
\hat{T}_{i}=T_{n-i}^{-1} \quad \text { and } \quad \hat{\omega}=\omega^{-1}
$$


have been used in applying the operation ^ to (1.28), while the second equality can be verified by substituting for $Y_{i}^{-1}$ using (1.15) and for $D_{i}$ using (1.28) and comparing with the first equality.

Since the $D_{i}$ commute, it follows from the definition of $\mathcal{D}_{i}$ that the $\left\{\mathcal{D}_{i}\right\}$ also form a commuting set. Moreover, using (2.4), one can check that the operators $\mathcal{D}_{i}$ possess the same relations with the generators $T_{i}, \omega$ as do the $D_{i}$, namely

$$
\begin{aligned}
T_{i} \mathcal{D}_{i+1} & =t \mathcal{D}_{i} T_{i}^{-1}, & T_{i} \mathcal{D}_{i}=\mathcal{D}_{i+1} T_{i}+(t-1) \mathcal{D}_{i}, & & 1 \leq i \leq n-1 \\
{\left[T_{i}, \mathcal{D}_{j}\right] } & =0, & & & j \neq i, i+1 \\
\mathcal{D}_{n} \omega & =q \omega \mathcal{D}_{1}, & \mathcal{D}_{i} \omega=\omega \mathcal{D}_{i+1} & & 1 \leq i \leq n-1 .
\end{aligned}
$$

To conclude the preliminaries, we follow Sahi [25] and introduce the generalized arm and leg (co-)lengths for a node $s \in \eta$ via

$$
\begin{array}{ll}
a(s)=\eta_{i}-j & l(s)=\#\left\{k>i \mid j \leq \eta_{k} \leq \eta_{i}\right\}+\#\left\{k<i \mid j \leq \eta_{k}+1 \leq \eta_{i}\right\} \\
a^{\prime}(s)=j-1 & l^{\prime}(s)=\#\left\{k>i \mid \eta_{k}>\eta_{i}\right\}+\#\left\{k<i \mid \eta_{k} \geq \eta_{i}\right\}
\end{array}
$$

and define the quantities

$$
\begin{array}{rlrl}
d_{\eta}(q, t) & :=\prod_{s \in \eta}\left(1-q^{a(s)+1} t^{l(s)+1}\right) & l(\eta):=\sum_{s \in \eta} l(s) \\
d_{\eta}^{\prime}(q, t):=\prod_{s \in \eta}\left(1-q^{a(s)+1} t^{l(s)}\right) & l^{\prime}(\eta):=\sum_{s \in \eta} l^{\prime}(s) \\
e_{\eta}(q, t):=\prod_{s \in \eta}\left(1-q^{a^{\prime}(s)+1} t^{n-l^{\prime}(s)}\right) & a(\eta):=\sum_{s \in \eta} a(s) .
\end{array}
$$

The statistics $l(\eta), l^{\prime}(\eta)$ and $a(\eta)$ generalize the quantity

$$
b(\lambda):=\sum_{i}(i-1) \lambda_{i}=\sum_{i}\left(\begin{array}{c}
\lambda_{i}^{\prime} \\
2
\end{array}\right)
$$

from partitions to compositions. From [25] these quantities have the following properties

Lemma 2.1. Let $\Phi \eta:=\left(\eta_{2}, \ldots, \eta_{n}, \eta_{1}+1\right)$. We have

$$
\begin{aligned}
& \frac{d_{\Phi \eta}(q, t)}{d_{\eta}(q, t)}=\frac{e_{\Phi \eta}(q, t)}{e_{\eta}(q, t)}=1-q t^{n+\bar{\eta}_{1}}, \quad \frac{d_{\Phi \eta}^{\prime}(q, t)}{d_{\eta}^{\prime}(q, t)}=1-q t^{n-1+\bar{\eta}_{1}}, \\
& \frac{e_{s_{i} \eta}(q, t)=e_{\eta}(q, t),}{d_{s_{i} \eta}(q, t)}=\frac{1-t^{\delta_{i, \eta}+1}}{1-t^{\delta_{i, \eta}}}, \quad \frac{d_{s_{i} \eta}^{\prime}(q, t)}{d_{\eta}^{\prime}(q, t)}=\frac{1-t^{\delta_{i, \eta}}}{1-t^{\delta_{i, \eta}-1}} \\
& \text { for } \eta_{i}>\eta_{i+1}, \quad \delta_{i, \eta}:=\bar{\eta}_{i}-\bar{\eta}_{i+1} \\
& a(\Phi \eta)=\eta_{1}+a(\eta), \quad l(\Phi \eta)=l(\eta)+\#\left\{k>1 \mid \eta_{k} \leq \eta_{1}\right\}
\end{aligned}
$$




$$
\begin{aligned}
& l^{\prime}(\Phi \eta)=l^{\prime}(\eta)+n-1-\#\left\{k>1 \mid \eta_{k} \leq \eta_{1}\right\} \\
& a\left(s_{i} \eta\right)=a(\eta) \quad l^{\prime}\left(s_{i} \eta\right)=l^{\prime}(\eta) \quad l\left(s_{i} \eta\right)=l(\eta)+1 \quad \text { for } \eta_{i}>\eta_{i+1} .
\end{aligned}
$$

A consequence of the first two equations in the final line is that

$$
l^{\prime}(\eta)=l^{\prime}\left(\eta^{+}\right)=b\left(\eta^{+}\right), \quad a(\eta)=a\left(\eta^{+}\right)=b\left(\left(\eta^{+}\right)^{\prime}\right)
$$

where $\left(\eta^{+}\right)^{\prime}$ denotes the partition conjugate to $\eta^{+}$.

\subsection{Raising Operators and Lowering Operators.}

There are two distinct raising operators which have a very simple action on non-symmetric Macdonald polynomials. Define $[\mathbf{1 3}, \mathbf{3}]$

$$
\begin{aligned}
& \Phi_{1}:=x_{n} \omega, \\
& \Phi_{2}:=x_{n} T_{n-1}^{-1} \cdots T_{2}^{-1} T_{1}^{-1} .
\end{aligned}
$$

A direct calculation reveals that for $i=1,2$

$$
\begin{array}{rlrl}
Y_{n} \Phi_{i} & =q \Phi_{i} Y_{1} & \\
Y_{j} \Phi_{i} & =\Phi_{i} Y_{j+1} & & 1 \leq j \leq n-1
\end{array}
$$

whence $\Phi_{i} E_{\eta}$ is a constant multiple of $E_{\Phi \eta}$, where $\Phi \eta:=\left(\eta_{2}, \ldots, \eta_{n}, \eta_{1}+1\right)$. This constant is determined by looking at the coefficient of $x^{\Phi \eta}$ with the result that

$$
\begin{aligned}
\Phi_{1} E_{\eta} & =q^{\eta_{1}} E_{\Phi \eta}, \\
\Phi_{2} E_{\eta} & =t^{-\#\left\{i \mid \eta_{i} \leq \eta_{1}\right\}} E_{\Phi \eta} .
\end{aligned}
$$

Remark. These operators are simply related via $\Phi_{1}=t^{n-1} \Phi_{2} Y_{1}$. Of course any function of the operators $Y_{i}$ multiplied by $\Phi_{1}$ will be a raising operator for the non-symmetric Macdonald polynomials but these two are in some sense the simplest.

In a similar manner, one can use the $q$-Dunkl operators (1.28) to construct lowering operators as follows,

$$
\begin{aligned}
& \Psi_{1}:=\omega^{-1} D_{n}, \\
& \Psi_{2}:=T_{1} T_{2} \cdots T_{n-1} D_{n} .
\end{aligned}
$$

$\Psi_{2}$ was introduced previously in $[3]$. These operators intertwine with the Cherednik operators as

$$
\begin{aligned}
& Y_{1} \Psi_{i}=q^{-1} \Psi_{i} Y_{n} \\
& Y_{j} \Psi_{i}=\Psi_{i} Y_{j-1} \quad 2 \leq j \leq n
\end{aligned}
$$

and it is seen that

$$
\begin{aligned}
& \Psi_{1} E_{\eta}=q^{-\eta_{n}+1}\left(1-t^{n-1+\bar{\eta}_{n}}\right) E_{\Psi \eta}, \\
& \Psi_{2} E_{\eta}=t^{\#\left\{i \mid \eta_{i}<\eta_{n}\right\}}\left(1-t^{n-1+\bar{\eta}_{n}}\right) E_{\Psi \eta}
\end{aligned}
$$

where $\Psi \eta:=\left(\eta_{n}-1, \eta_{1}, \ldots, \eta_{n-1}\right)$. 


\subsection{Kernel.}

Let $\sim$ denote the involution on the ring of polynomials with coefficients in $\mathbb{C}(q, t)$, which acts on the the coefficients by sending $q \mapsto q^{-1}, t \mapsto t^{-1}$, and extend it to act on operators in the obvious way. Define the kernel

$$
\mathcal{K}_{A}(x ; y ; q, t)=\sum_{\eta} q^{a(\eta)} t^{(n-1)|\eta|-l^{\prime}(\eta)} \frac{d_{\eta}}{d_{\eta}^{\prime} e_{\eta}} E_{\eta}(x) \widetilde{E}_{\eta}(y) .
$$

It follows from (2.7) that this kernel is related to the previously introduced kernel [3]

$$
K_{A}(x ; y ; q, t)=\sum_{\eta} \frac{d_{\eta}}{d_{\eta}^{\prime} e_{\eta}} E_{\eta}(x) \widetilde{E}_{\eta}(y)
$$

((2.13) was denoted by $\mathcal{K}_{A}$ in [3], but for the present purpose it is desirable to use this notation for (2.12)) by means of

$$
\widetilde{\mathcal{K}}_{A}(x ; y ; q, t)=K_{A}(-q y ; x ; q, t) .
$$

The kernel $\mathcal{K}_{A}(x ; y ; q, t)$ satisfies the following properties:

\section{Theorem 2.2.}

$$
\begin{array}{ll}
\text { (a) } & \left(T_{i}^{ \pm 1}\right)^{(x)} \mathcal{K}_{A}(x ; y ; q, t)=\left(\widetilde{T_{i}^{\mp 1}}\right)^{(y)} \mathcal{K}_{A}(x ; y ; q, t) \\
\text { (b) } & \Psi_{1}^{(x)} \mathcal{K}_{A}(x ; y ; q, t)=\widetilde{\Phi_{2}}{ }^{(y)} \mathcal{K}_{A}(x ; y ; q, t) \\
\text { (c) } & \mathcal{D}_{i}^{(x)} \mathcal{K}_{A}(x ; y ; q, t)=y_{i} \mathcal{K}_{A}(x ; y ; q, t) .
\end{array}
$$

Proof. The proof of this result follows the same line of reason as in $[\mathbf{3}$, Thm. $5.2]$, using the facts that

$$
\begin{aligned}
x_{i} & =t^{-n+i} \widetilde{T_{i}^{-1}} \cdots \widetilde{T_{n-1}^{-1}} \widetilde{\Phi_{2}} \widetilde{T_{1}} \cdots \widetilde{T_{i-1}} \\
\mathcal{D}_{i} & =t^{-n+i} T_{i-1}^{-1} \cdots T_{1}^{-1} \Psi_{1} T_{n-1} \cdots T_{i} .
\end{aligned}
$$

We recall from [3] that the analogue of property (c) for the kernel $K_{A}(x ; y ; q, t)$ is

$$
D_{i}^{(x)} K_{A}(x ; y ; q, t)=y_{i} K_{A}(x ; y ; q, t) .
$$

A feature of both property (c) and (2.17) is that the $q$-Dunkl operator $\mathcal{D}_{i}$ (resp. $D_{i}$ ) act on the left set of variables only. However, by applying the operation $\sim$ and using (2.14), we can form similar identities where they act on the right set of variables, namely:

\section{Corollary 2.3.}

$$
\begin{aligned}
& \left(\widetilde{\mathcal{D}_{i}}\right)^{(x)} K_{A}(z ; x ; q, t)=-q^{-1} z_{i} K_{A}(z ; x ; q, t) \\
& \left(\widetilde{D_{i}}\right)^{(y)} \mathcal{K}_{A}(x ; y ; q, t)=-q x_{i} \mathcal{K}_{A}(x ; y ; q, t) .
\end{aligned}
$$




\subsection{First isomorphism.}

Returning to the proof of Thm. 1.1, we claim that it follows from the subsequent

Proposition 2.4. Let $\mathcal{R}_{q, t}$ be the subalgebra of the algebra of endomorphism on the polynomial ring $\mathbb{Q}(q, t)\left[x_{1}, \ldots, x_{n}\right]$ generated by the elements $\left\{T_{i}, \omega, x_{i}\right\}$ with relations given by (1.11)-(1.14), (2.1) The map $\phi: \mathcal{R}_{q^{-1}, t^{-1}}$ $\longrightarrow \mathcal{R}_{q, t}$ defined by

$$
\phi\left(\widetilde{\omega^{-1}}\right)=T_{1} \cdots T_{n-1} \omega T_{1}^{-1} \cdots T_{n-1}^{-1}, \quad \phi\left(x_{i}\right)=e_{i}, \quad \phi\left(\widetilde{T_{i}^{ \pm 1}}\right)=T_{i}^{\mp 1}
$$

is an algebra isomorphism.

Proof. Note that a simple consequence of the definition of $\phi$ given above, is the relation

$$
\phi\left(\widetilde{Y_{i}^{-1}}\right)=Y_{i} .
$$

The proof that $\phi$ is indeed an isomorphism follows by a standard calculation.

Proof of Thm. 1.1. We know that $E_{\eta}\left(x ; q^{-1}, t^{-1}\right)$ is an eigenfunction of $\widetilde{Y_{i}^{-1}}$. This is shown by utilizing the relations amongst the operators $\left\{\tilde{Y}_{i}^{-1}\right.$, $\left.\tilde{T}_{i}^{ \pm 1}, x_{i}\right\}$ to move the operators $\widetilde{Y_{i}^{-1}}$ through the terms in $E_{\eta}\left(x ; q^{-1}, t^{-1}\right)$, until one obtains $\widetilde{Y_{i}^{-1}} \cdot 1=t^{-n+1} \cdot 1$. By adopting this viewpoint in the eigenvalue equation (considered as an identity in $\mathcal{R}_{q^{-1}, t^{-1}}$ )

$$
\widetilde{Y_{i}^{-1}} E_{\eta}\left(x ; q^{-1}, t^{-1}\right) \cdot 1=t^{\bar{\eta}_{i}} E_{\eta}\left(x ; q^{-1}, t^{-1}\right) \cdot 1
$$

and applying the map $\phi$ to both sides it then follows from (2.20), (2.21) that $E_{\eta}\left(e ; q^{-1}, t^{-1}\right) \cdot 1$ is an eigenfunction of $\phi\left(\widetilde{Y_{i}^{-1}}\right)=Y_{i}$, with leading order term $x^{\eta}$ and hence must be proportional to $E_{\eta}(x ; q, t)$.

To determine the proportionality constant $\alpha_{\eta}(q, t)$ say, it follows from the action of $T_{i}$ given by (2.2) that

$$
e_{1}^{\eta_{1}} e_{2}^{\eta_{2}} \cdots e_{n}^{\eta_{n}} \cdot 1=q^{f(\eta)} t^{g(\eta)} x^{\eta}+\sum_{\nu<\eta} b_{\eta \nu} x^{\nu}
$$

where $f(\eta)=\sum_{i}\left(\begin{array}{c}\eta_{i} \\ 2\end{array}\right)$ and

$$
\begin{aligned}
& g(q)=\sum_{i=1}^{n}\left(\eta_{i}-1\right)(i-1)+\sum_{i=0}^{\eta_{n-1}} \chi\left(\eta_{n} \leq i\right)+\sum_{i=0}^{\eta_{n-2}} \chi\left(\eta_{n} \leq i\right)+\chi\left(\eta_{n-1} \leq i\right) \\
& (2.22) \quad+\cdots+\sum_{i=0}^{\eta_{1}} \chi\left(\eta_{n} \leq i\right)+\cdots+\chi\left(\eta_{2} \leq i\right)
\end{aligned}
$$

where $\chi(P)=1$ if $P$ is true, and zero otherwise. The simplification $g(q)=$ $\sum_{i}(n-i) \eta_{i}^{+}-\ell\left(w_{\eta}\right)$ then follows from the above expression by induction on $\ell\left(w_{\eta}\right)$. 


\section{Al-Salam \& Carlitz polynomials.}

The isomorphism $\phi$ introduced in the previous section can be generalized to another isomorphism $\psi_{a}$ such that $\psi_{a}\left(x_{i}\right)$ includes not just degree-raising parts, but degree-preserving and lowering parts as well. It will turn out that this isomorphism is precisely what is needed to obtain non-symmetric analogues of the Al-Salam\&Carlitz polynomials in the same way as was done for the Hermite case.

As previously mentioned, the symmetric ASC polynomials $V_{\lambda}^{(a)}$ can be defined via their orthogonality with respect to the inner product (1.20). We remark that under this inner product we have the important result that the adjoint operators of $T_{i}^{ \pm 1}, \omega$ are given by

$$
\left(T_{i}^{ \pm 1}\right)^{*}=T_{i}^{ \pm 1}, \quad\left(\omega^{-1}\right)^{*}=\frac{t^{n-1}}{a q} \omega\left(x_{1}-q\right)\left(x_{1}-a q\right) .
$$

The ASC polynomials $V_{\lambda}^{(a)}$ can equivalently be defined by means of the generating function $[\mathbf{1}]$

$$
\prod_{i=1}^{n} \frac{1}{\rho_{a}\left(t^{-(n-1)} x_{i} ; q\right)}{ }_{0} \psi_{0}(x ; y ; q, t)=\sum_{\lambda} \frac{(-1)^{|\lambda|} q^{b\left(\lambda^{\prime}\right)} V_{\lambda}^{(a)}(y ; q, t) P_{\lambda}(x ; q, t)}{d_{\lambda}^{\prime}(q, t) P_{\lambda}\left(1, t, \ldots, t^{n-1} ; q, t\right)} .
$$

Here, $\rho_{a}(x):=(x ; q)_{\infty}(a x ; q)_{\infty}, b(\lambda)$ is defined by $(2.8)$ and

$$
\begin{aligned}
P_{\lambda}\left(1, t, \ldots, t^{n-1} ; q, t\right) & =t^{l(\lambda)} \prod_{s \in \lambda} \frac{\left(1-q^{a^{\prime}(s)} t^{n-l^{\prime}(s)}\right)}{\left(1-q^{a(s)} t^{l(s)+1}\right)} \\
{ }_{0} \psi_{0}(x ; y ; q, t) & :=\sum_{\lambda} \frac{(-1)^{|\lambda|} q^{b(\lambda)}}{d_{\lambda}^{\prime}(q, t) P_{\lambda}\left(1, t, \ldots, t^{n-1} ; q, t\right)} P_{\lambda}(x ; q, t) P_{\lambda}(y ; q, t) .
\end{aligned}
$$

This latter kernel was previously introduced by Kaneko [11] in connection with hypergeometric solutions of systems of $q$-difference equations.

Similarly the ASC polynomials $U_{\kappa}^{(a)}$ can be defined by the generating function [1]

$$
\rho_{a}\left(x_{1} ; q\right) \cdots \rho_{a}\left(x_{n} ; q\right){ }_{0} \mathcal{F}_{0}(x ; y ; q, t)=\sum_{\kappa} \frac{t^{b(\kappa)} U_{\kappa}^{(a)}(y ; q, t) P_{\kappa}(x ; q, t)}{d_{\kappa}^{\prime}(q, t) P_{\kappa}\left(1, t, \ldots, t^{n-1} ; q, t\right)}
$$

where the hypergeometric function ${ }_{0} \mathcal{F}_{0}$ is defined by

$$
{ }_{0} \mathcal{F}_{0}(x ; y ; q, t):=\sum_{\kappa} \frac{t^{b(\kappa)}}{d_{\kappa}^{\prime}(q, t) P\left(1, t, \ldots, t^{n-1} ; q, t\right)} P_{\kappa}(x ; q, t) P_{\kappa}(y ; q, t) .
$$




\subsection{Second isomorphism.}

Consider the involution ' on polynomials and operators defined in the statement of Corollary 1.3. The operator $E_{i}$ introduced in (1.29) has its origins in this involution, namely,

$$
E_{i}:=\left(\hat{D}_{n+1-i}\right)^{*}:=\left(-\frac{1}{q} \mathcal{D}_{i}\right)^{*} .
$$

The form (1.29) follows from (3.5) by making use of the adjoint formulae (3.1). The relations between the operators $E_{i}$ and the operators $\left\{D_{i}, T_{i}, \omega\right\}$, can be derived using (3.5). Thus, for example, application of the adjoint operation ${ }^{*}$ to the relations involving $\mathcal{D}_{i}, T_{i}$ gives, in place of the first relation in (2.5),

$$
T_{i}^{-1} E_{i} T_{i}^{-1}=t^{-1} E_{i+1}
$$

Now consider the following mapping $\psi_{a}:\left\{\widetilde{\omega}^{-1}, \widetilde{T}_{i}, x_{i}, \widetilde{\mathcal{D}}_{i}\right\} \longrightarrow\left\{\omega, T_{i}, x_{i}, d_{i}\right\}$ where each set of operators defines a certain algebra of endomorphisms on the ring $\mathbb{Q}(q, t)\left[x_{1}, \ldots, x_{n}\right]$, defined by

$$
\begin{aligned}
\psi_{a}\left(x_{i}\right) & =E_{i}, \\
\psi_{a}\left(\widetilde{\omega}^{-1}\right) & =T_{1} \cdots T_{n-1}\left(Y_{n}+(1+a) t^{1-n} D_{n}+a t^{2-2 n} D_{n} Y_{n} D_{n}\right), \\
\psi_{a}\left(\widetilde{T}_{i}^{-1}\right) & =T_{i}, \\
\psi_{a}\left(\widetilde{\mathcal{D}}_{i}\right) & =-a t^{n+1-2 i} T_{i-1} \cdots T_{1} T_{1} \cdots T_{i-1} E_{i}^{*} T_{i}^{-1} \cdots T_{n-1}^{-1} T_{n-1}^{-1} \cdots T_{i}^{-1} .
\end{aligned}
$$

Then Theorem 1.2 will follow from:

Proposition 3.1. The map $\psi_{a}$ is an algebra isomorphism.

Proof. The proof of this result consists of checking that the operators $\psi_{a}(u)$ given in (3.7) satisfy the same relations as the original operators $u$, given by (1.11)-(1.14), (2.1) and (2.5), (after application of the involution $\sim)$. For example, the first formula in (2.1), after application of the involution , reads

$$
\tilde{T}_{i}^{-1} x_{i+1}=t x_{i} \tilde{T}_{i}
$$

Now applying the mapping $\psi_{a}$ gives

$$
T_{i} E_{i+1}=t E_{i} T_{i}^{-1} \text {. }
$$

But this is equivalent to (3.6) so the algebra is indeed preserved. The calculations involved in checking the other relations are typically more involved; however they are similar to those undertaken in [3], and so for brevity will be omitted.

As with the relationship between Prop. 2.4 and the proof of Thm. 1.1 we are in a position to complete the: 
Proof of Thm. 1.2. From Thm. 1.1, and the definition (1.29) of the operators $E_{i}$ it follows that $E_{\eta}^{(V)}$ has leading term $E_{\eta}(x ; q, t)$. In addition, it follows from (3.7) that

$$
\psi_{a}\left(\widetilde{Y_{i}^{-1}}\right)=Y_{i}+(1+a) t^{1-n} D_{i}+a t^{2-2 n} D_{i} Y_{i}^{-1} D_{i}
$$

and from Prop. 3.1, that these are eigenoperators for the non-symmetric ASC polynomials defined by (1.30). The corresponding eigenvalue is simply $t^{\bar{\eta}_{i}}$. By writing these operators out explicitly, it is seen that they are selfadjoint w.r.t. the inner product (1.20). Hence by standard arguments, the polynomials (1.30) are orthogonal w.r.t. (1.20).

\subsection{Normalization.}

The images of the raising and lowering operators (2.10), (2.11) (after application of ) under the map $\psi_{a}$ are guaranteed, by virtue of Prop. 3.1, to be raising and lowering operators for the polynomials $E_{\eta}^{(V)}(x)$.

In particular, using (2.16) and (3.7) we see that

$$
\psi_{a}\left(\widetilde{\Psi_{1}}\right)=a q^{-1} t^{1-n} \Psi_{1}
$$

so that $\Psi_{1}$ remains a raising operator for the polynomials $E_{\eta}^{(V)}$. By examination of the leading terms, we must have

$$
\Psi_{1} E_{\eta}^{(V)}=q^{\eta_{n}+1} \frac{d_{\eta}^{\prime}}{d_{\Psi \eta}^{\prime}} E_{\Psi \eta}^{(V)} .
$$

Also, use of (2.15) and (3.7) gives

$$
\psi_{a}\left(\widetilde{\Phi_{2}}\right)=-q^{-1} \Psi_{1}^{*}
$$

so that $\Psi_{1}^{*}$ is a raising operator for $E_{\eta}^{(V)}$. Indeed,

$$
\Psi_{1}^{*} E_{\eta}^{(V)}=a^{-1} t^{n-1} q^{\eta_{1}+1} E_{\Phi \eta}^{(V)} .
$$

By an argument similar to that used in [4, Prop. 3.6] it follows from (3.9) and (3.10) that

$$
\left\langle E_{\Phi \eta}^{(V)}, E_{\Phi \eta}^{(V)}\right\rangle^{(V)}=a t^{1-n} q^{-2 \eta_{1}-1} \frac{d_{\Phi \eta}^{\prime}}{d_{\eta}^{\prime}}\left\langle E_{\eta}^{(V)}, E_{\eta}^{(V)}\right\rangle^{(V)}
$$

Also, we have

$$
\left\langle E_{s_{i} \eta}^{(V)}, E_{s_{i} \eta}^{(V)}\right\rangle^{(V)}=\frac{\left(1-t^{\delta_{i \eta}-1}\right)\left(1-t^{\delta_{i \eta}+1}\right)}{t\left(1-t^{\delta_{i \eta}}\right)^{2}}\left\langle E_{\eta}^{(V)}, E_{\eta}^{(V)}\right\rangle^{(V)} .
$$

The solution of the recurrence relations (3.11), (3.12) gives:

\section{Proposition 3.2.}

$$
\mathcal{N}_{\eta}^{(V)}:=\left\langle E_{\eta}^{(V)}, E_{\eta}^{(V)}\right\rangle^{(V)}=\left(a q^{-1} t^{2-2 n}\right)^{|\eta|} q^{-2 a(\eta)} t^{l(\eta)+l^{\prime}(\eta)} \frac{d_{\eta}^{\prime} e_{\eta}}{d_{\eta}} \mathcal{N}_{0}^{(V)}
$$


where for $t=q^{k},[\mathbf{1}]$

$$
\mathcal{N}_{0}^{(V)}=(1-q)^{n} a^{k n(n-1) / 2} t^{-2 k\left(\begin{array}{c}
n \\
3
\end{array}\right)-k\left(\begin{array}{c}
n \\
2
\end{array}\right)} \prod_{l=1}^{n} \frac{(q ; q)_{k l}}{(q ; q)_{k}} .
$$

By using the formulas (1.32), (1.26) and (1.27) we see that the norm $\mathcal{N}_{\eta}^{(U)}$ of the non-symmetric ASC polynomials $E_{\eta}^{(U)}$ with respect to the inner product (1.23) is given by simply replacing $q, t$ by $q^{-1}, t^{-1}$ in (3.13). Use of (2.7) then gives:

\section{Corollary 3.3.}

$$
\mathcal{N}_{\eta}^{(U)}:=\left\langle E_{\eta}^{(U)}, E_{\eta}^{(U)}\right\rangle^{(U)}=\left(a t^{n-1}\right)^{|\eta|} q^{a(\eta)} t^{-l(\eta)} \frac{d_{\eta}^{\prime} e_{\eta}}{d_{\eta}} \mathcal{N}_{0}^{(U)}
$$

where for $t=q^{k},[\mathbf{1}]$

$$
\mathcal{N}_{0}^{(U)}=(1-q)^{n}(-a)^{k n(n-1) / 2} t^{k\left(\begin{array}{c}
n \\
3
\end{array}\right)-\frac{k-1}{2}\left(\begin{array}{c}
n \\
2
\end{array}\right)} \prod_{l=1}^{n} \frac{(q ; q)_{k l}}{(q ; q)_{k}} .
$$

\subsection{Generating function.}

The raising operator expression (1.30) facilitates the derivation of the generating function for the non-symmetric ASC polynomials. Also required will be the $q$-symmetrization of (2.12).

Proposition 3.4. Let [18] $U^{+}=\sum_{\sigma} T_{\sigma}$ where $T_{\sigma}:=T_{i_{1}} \cdots T_{i_{p}}$ for a reduced word decomposition $\sigma=s_{i_{1}} \cdots s_{i_{p}}$. We have

$$
\left(U^{+}\right)^{(x)} \mathcal{K}_{A}(x ; y ; q, t)=[n]_{t} !_{0} \psi_{0}\left(x ;-t^{n-1} y ; q, t\right)
$$

where ${ }_{0} \psi_{0}$ is defined by (3.2).

Proof. We remark that this is the analogue of the result [3, Prop. 5.4]

$$
\left(U^{+}\right)^{(x)} K_{A}(x ; y ; q, t)=[n]_{t} !_{0} F_{0}(x ; y ; q, t) .
$$

In fact in our proof of (3.15) we will use the formula

$$
U^{+} E_{\eta}(x)=[n]_{t} ! t^{l(\eta)} \frac{e_{\eta}}{P_{\lambda}\left(t^{\delta}\right) d_{\eta}} P_{\lambda}(x), \quad \lambda=\eta^{+}
$$

which was deduced [3, eqs. (5.8)\&(5.18)] as a corollary of (3.16). Thus we apply $U^{+}$to $(2.12)$ and use (3.17) to compute its action. Simplifying the result using the first equation in (2.9) and the formula [18]

$$
P_{\lambda}(y)=\sum_{\eta: \eta^{+}=\lambda} \frac{d_{\lambda}^{\prime}}{d_{\eta}^{\prime}} E_{\eta}(y)
$$

the result then follows. 
Consider now the generating function

$$
F_{1}(y ; z)=\sum_{\nu} A_{\nu} E_{\nu}^{(V)}(y) \widetilde{E}_{\nu}(z)
$$

where

$$
A_{\nu}=(a / q)^{|\nu|} \frac{\mathcal{N}_{0}^{(V)}}{\alpha_{\nu}(q, t) \mathcal{N}_{\nu}^{(V)}}=q^{a(\nu)} t^{(n-1)|\nu|-l^{\prime}(\nu)} \frac{d_{\nu}}{d_{\nu}^{\prime} e_{\nu}} .
$$

Here we have used the fact that $l(\eta)=l\left(\eta^{+}\right)+\ell\left(w_{\eta}\right)$ to rewrite $\alpha_{\eta}(q, t)$ as defined by (1.18) as

$$
\alpha_{\eta}(q, t)=q^{a(\eta)} t^{(n-1)|\eta|-l(\eta)}
$$

Clearly

$$
\left\langle F_{1}(y ; z), E_{\eta}^{(V)}(y)\right\rangle_{y}^{(V)}=(a / q)^{|\eta|} \frac{\mathcal{N}_{0}^{(V)}}{\alpha_{\eta}(q, t)} \widetilde{E}_{\eta}(z) .
$$

Next note the integration formula

$$
\begin{aligned}
\left\langle\mathcal{K}_{A}(y ; z), 1\right\rangle_{y}^{(V)} & =\frac{1}{[n]_{t} !}\left\langle U_{y}^{+} \mathcal{K}_{A}(y ; z), 1\right\rangle_{y}^{(V)} \\
& =\left\langle{ }_{0} \psi_{0}\left(y ;-t^{n-1} z\right), 1\right\rangle_{y}^{(V)}=\mathcal{N}_{0}^{(V)} \prod_{i=1}^{n} \rho_{a}\left(-z_{i}\right)
\end{aligned}
$$

which follows from the symmetrization formula (3.15), the fact that $U_{y}^{+}$is self adjoint w.r.t. $\langle,\rangle_{y}^{(V)}$ and an integral formula for the kernel ${ }_{0} \psi_{0}(y ; z)$ given in $[\mathbf{1}$, Prop 4.8], and consider the generating function

$$
F_{2}(y ; z)=\prod_{i=1}^{n} \frac{1}{\rho_{a}\left(-z_{i}\right)} \mathcal{K}(y ; z) .
$$

We have

$$
\begin{aligned}
\left\langle F_{2}(y ; z), E_{\eta}^{(V)}(y)\right\rangle_{y}^{(V)} & =\frac{(-a)^{|\eta|}}{\alpha_{\eta}(q, t)} \prod_{i} \frac{1}{\rho_{a}\left(-z_{i}\right)}\left\langle\mathcal{K}(y ; z), \widetilde{E}_{\eta}\left(E^{(y)}\right)\right\rangle_{y}^{(V)} \\
& =\frac{(a / q)^{|\eta|}}{\alpha_{\eta}(q, t)} \prod_{i} \frac{1}{\rho_{a}\left(-z_{i}\right)}\left\langle\widetilde{E}_{\eta}\left(\mathcal{D}^{(y)}\right) \mathcal{K}(y ; z), 1\right\rangle_{y}^{(V)} \\
& =\frac{(a / q)^{|\eta|}}{\alpha_{\eta}(q, t)} \prod_{i} \frac{1}{\rho_{a}\left(-z_{i}\right)} \widetilde{E}_{\eta}(z)\langle\mathcal{K}(y ; z), 1\rangle_{y}^{(V)} \\
& =(a / q)^{|\eta|} \frac{\mathcal{N}_{0}^{(V)}}{\alpha_{\eta}(q, t)} \widetilde{E}_{\eta}(z) .
\end{aligned}
$$

In the above chain of equalities, we have used (1.30), (3.5), the kernel property Thm. 2.2 (c) and (3.20) respectively. The non-symmetric ASC polynomials $E_{\eta}^{(V)}(y)$ are a complete basis for polynomials in $y$ and hence from 
above we have $F_{1}=F_{2}$. That is, we have the generating function for nonsymmetric ASC polynomials $E_{\nu}^{(V)}$.

Proposition 3.5. With $A_{\nu}$ given by (3.19)

$$
\prod_{i=1}^{n} \frac{1}{\rho_{a}\left(-z_{i}\right)} \mathcal{K}_{A}(y ; z)=\sum_{\nu} A_{\nu} E_{\nu}^{(V)}(y) \widetilde{E}_{\nu}(z) .
$$

We remark that this generating function could also be derived in a manner similar to that used in the symmetric case [1], namely by applying the operator $\left.\widetilde{\left(Y_{i}^{-1}\right.}\right)^{(z)}$ to both sides of $(3.21)$ and deducing that $E_{\eta}^{(V)}(y)$ is an eigenfunction of

$$
h_{i}=\psi_{a}\left(\widetilde{Y_{i}^{-1}}\right)=Y_{i} T_{i-1} \cdots T_{1}\left(1+\mathcal{D}_{1}\right)\left(1+a \mathcal{D}_{1}\right) T_{1}^{-1} \cdots T_{i-1}^{-1}
$$

with leading term $E_{\eta}(y)$ (some manipulation using (2.5) and (2.3) casts this into the form given in (1.31)). Note also that by applying the operation with the respect to the $y$-variables in (3.21) and using the formula (2.14) as well as

$$
\left.\frac{1}{\rho_{a}(x ; q)}\right|_{q \mapsto q^{-1}}=\rho_{a}(q x ; q),
$$

(see e.g. [1]) we deduce the generating function formula for the polynomials $E_{\nu}^{(U)}$.

\section{Corollary 3.6.}

$$
\prod_{i=1}^{n} \rho_{a}\left(z_{i}\right) K_{A}\left(z ; y^{R} ; q, t\right)=\sum_{\nu} \frac{d_{\nu}}{d_{\nu}^{\prime} e_{\nu}} E_{\nu}^{(U)}(y) E_{\nu}(z) .
$$

The generating function formulas in turn imply a further class of operator formulas relating the ASC polynomials and the non-symmetric Jack polynomials (c.f. [1, eqs. (3.9)\&(3.10)]).

Corollary 3.7. We have

$$
\begin{aligned}
& E_{\eta}^{(V)}(y)=\prod_{i=1}^{n} \frac{1}{\rho_{a}\left(-\mathcal{D}_{i}^{(y)}\right)} E_{\eta}(y) \\
& E_{\eta}^{(U)}(y)=\prod_{i=1}^{n} \rho_{a}\left(-q \widetilde{\mathcal{D}_{i}^{(y)}}\right) \widetilde{E}_{\eta}\left(y^{R}\right) .
\end{aligned}
$$

Proof. The first identity follows from (3.21) by using Thm. 2.2 (c) and comparing coefficients of $\widetilde{E}_{\eta}(z)$, while the second identity follows similarly from (3.23) and (2.18).

As further applications of the generating functions we will present some evaluation formulas for $E_{\eta}^{(V)}$ at the special points $t^{\bar{\delta}-n+1}$ and $a t^{\bar{\delta}-n+1}$, where $t^{\bar{\delta}}:=\left(1, t, t^{2}, \ldots, t^{n-1}\right)$. 
Proposition 3.8. We have

$$
\begin{aligned}
E_{\eta}^{(V)}\left(t^{\bar{\delta}-n+1}\right) & =(-a)^{|\eta|} q^{-a(\eta)} t^{l^{\prime}(\eta)-(n-1)|\eta|} E_{\eta}\left(t^{\bar{\delta}}\right) \\
E_{\eta}^{(V)}\left(a t^{\bar{\delta}-n+1}\right) & =(-1)^{|\eta|} q^{-a(\eta)} t^{l^{\prime}(\eta)-(n-1)|\eta|} E_{\eta}\left(t^{\bar{\delta}}\right)
\end{aligned}
$$

where

$$
E_{\eta}\left(t^{\bar{\delta}}\right)=t^{l(\eta)} \frac{e_{\eta}}{d_{\eta}}
$$

Proof. The formula (3.28) is a special case of a result of Cherednik [7] (see also [20]). For the derivation of (3.26) and (3.27) we follow the strategy of the proof of the analogous result in the symmetric case [1, Prop. 4.3]. First, note from the definition (1.8) that in general

$$
T_{i} f\left(t^{\bar{\delta}}\right)=t f\left(t^{\bar{\delta}}\right)
$$

and so

$$
\left(U^{+} f\right)\left(t^{\bar{\delta}}\right)=\left(U^{+} 1\right) f\left(t^{\bar{\delta}}\right)=[n]_{t} ! f\left(t^{\bar{\delta}}\right) .
$$

Use of this latter formula in (3.15) with $y=t^{\bar{\delta}}$ gives

$$
\mathcal{K}_{A}\left(t^{\bar{\delta}} ; z ; q, t\right)={ }_{0} \psi_{0}\left(t^{\bar{\delta}} ;-t^{n-1} z ; q, t\right)=\prod_{i=1}^{n}\left(-t^{n-1} z_{i} ; q\right)_{\infty},
$$

and similarly, from (3.16)

$$
K_{A}\left(t^{\bar{\delta}} ; z ; q, t\right)={ }_{0} F_{0}\left(t^{\bar{\delta}} ; z ; q, t\right)=\frac{1}{\prod_{i=1}^{n}\left(z_{i} ; q\right)_{\infty}},
$$

where the final equalities in (3.29) and (3.30) are known results $[\mathbf{1 7}, \mathbf{1 2}]$. Now set $y=t^{\bar{\delta}-n+1}$ in the generating function (3.15). Use of (3.29) with $z$ replaced by $t^{-n+1} z$, and then use of (3.30) allows the l.h.s. of the resulting expression to be written

$$
\frac{1}{\prod_{i=1}^{n}\left(-a z_{i} ; q\right)_{\infty}}=K_{A}\left(t^{\bar{\delta}} ;-a z ; q, t\right)=\sum_{\eta} \frac{(-a)^{|\eta|} d_{\eta}}{d_{\eta}^{\prime} e_{\eta}} E_{\eta}\left(t^{\bar{\delta}}\right) \widetilde{E}_{\eta}(z) .
$$

Equating with $\widetilde{E}_{\eta}(z)$ on the r.h.s. of the resulting expression gives (3.26). The formula (3.27) follows similarly, by substituting $y=a t^{\bar{\delta}-n+1}$ in (3.15).

\subsection{Relationship to the symmetric ASC polynomials.}

The non-symmetric ASC polynomials are related to the corresponding symmetric ASC polynomials in an analogous way to the relationship (3.17) between the non-symmetric and symmetric Macdonald polynomials.

Proposition 3.9. Let

$$
a_{\eta}(q, t)=[n]_{t} ! t^{\ell(\eta)} \frac{e_{\eta}}{P_{\eta^{+}}\left(t^{\bar{\delta}}\right) d_{\eta}} .
$$


We have

$$
\begin{aligned}
U^{+} E_{\eta}^{(V)}(y) & =a_{\eta}(q, t) V_{\eta^{+}}^{(a)}(y ; q, t) \\
U^{+} E_{\eta}^{(U)}(y) & =a_{\eta}(q, t) U_{\eta^{+}}^{(a)}(y ; q, t) .
\end{aligned}
$$

Proof. Consider the action of the $U^{+}$operator on (3.24) and (3.25). From the first three equations of (2.5) one can check that $T_{i}$ commutes with any symmetric function of the $\mathcal{D}_{i}$. Thus the action of $U^{+}$can be commuted to act to the right of $\prod_{i} \rho_{a}\left(-\frac{1}{q} \tilde{\mathcal{D}}_{i}\right)$ and $1 / \prod_{i} \rho_{a}\left(-\mathcal{D}_{i}\right)$. Use of $(3.17)$ then gives

$$
\begin{aligned}
U^{+} E_{\eta}^{(V)}(y) & =a_{\eta}(q, t) \frac{1}{\prod_{i} \rho_{a}\left(-\mathcal{D}_{i}\right)} P_{\eta^{+}}(y)=a_{\eta}(q, t) \frac{1}{\prod_{i} \rho_{a}\left(q \widetilde{D}_{i}\right)} P_{\eta^{+}}(y) \\
U^{+} E_{\eta}^{(U)}(y) & =a_{\eta}(q, t) \prod_{i} \rho_{a}\left(-q \widetilde{\mathcal{D}}_{i}\right) P_{\eta^{+}}(y)=a_{\eta}(q, t) \prod_{i} \rho_{a}\left(D_{i}\right) P_{\eta^{+}}(y),
\end{aligned}
$$

where in obtaining the first equality in the second formula we have used the fact that $\widetilde{P}_{\eta}\left(y^{R}\right)=P_{\eta}(y)$, while the second equalities in both formulas make use of (2.3) and the fact that $P_{\eta^{+}}$is a symmetric function. But the resulting operator formulas are precisely representations obtained in $[\mathbf{1}$, Eq. (3.9)\&(3.10)] for the symmetric ASC polynomials.

We can also relate the eigenoperators $h_{i}$ for the non-symmetric ASC polynomials $E_{\eta}^{(V)}$ to the eigenoperator [1, Eq. (3.28)]

$$
\begin{array}{r}
\mathcal{H}=t^{1-n} \sum_{i=1}^{n} Y_{i}^{-1}-(1+a) \sum_{i=1}^{n} t^{1-i} D_{i} Y_{i}^{-1}+a \sum_{i=1}^{n} t^{1-i} D_{i}^{2} Y_{i}^{-1} \\
+a\left(1-t^{-1}\right) \sum_{1 \leq i<j \leq n} t^{1-i} D_{j} D_{i} Y_{i}^{-1}
\end{array}
$$

for the symmetric ASC polynomials $U_{\lambda}^{(a)}$.

Proposition 3.10. Let $h_{i}$ be given by (1.31) and $\mathcal{H}$ by (3.33). When acting on symmetric functions

$$
\sum_{i=1}^{n} h_{i}=t^{1-n} \tilde{\mathcal{H}}
$$

Proof. From Theorem 1.2, by summing over $i$ in (1.31) we have

$$
\sum_{i=1}^{n} h_{i} E^{(V)}(x ; q, t)=t^{1-n} e\left(\eta^{+}\right) E^{(V)}(x ; q, t),
$$

where $e\left(\eta^{+}\right)=\sum_{i=1}^{n} t^{\bar{\eta}_{i}}=\sum_{i=1}^{n} q^{\eta_{i}^{+}} t^{n-i}$. We would next like to apply the operator $U^{+}$to both sides of this eigenvalue equation. For this purpose we require the fact that $T_{i}$ commutes with $\sum_{i=1}^{n} h_{i}$ (this follows from (1.16), 
and the fact that these same equations apply with the $Y_{i}$ replaced by $D_{i}$ ). Thus, making use of (3.31), this operation gives

$$
\sum_{i=1}^{n} h_{i} V_{\eta^{+}}^{(a)}(x ; q, t)=t^{1-n} e\left(\eta^{+}\right) V_{\eta^{+}}^{(a)}(x ; q, t) .
$$

But from [1] we know that this same eigenvalue equation applies with $\sum_{i=1}^{n} h_{i}$ replaced by $t^{1-n} \tilde{\mathcal{H}}$. The result now follows from the fact that $\left\{V_{\eta^{+}}^{(a)}\right\}$ are a basis for symmetric functions.

We remark that an alternative proof is to establish directly that when acting on symmetric functions

$$
\begin{aligned}
& \sum_{i=1}^{n} \tilde{Y}_{i}^{-1}=\sum_{i=1}^{n} Y_{i} \\
& -\sum_{i=1}^{n} t^{-1+i} \tilde{D}_{i} \tilde{Y}_{i}^{-1}=\sum_{i=1}^{n} D_{i} \\
& \sum_{i=1}^{n} t^{-1+i} \tilde{D}_{i}^{2} \tilde{Y}_{i}^{-1}+(1-t) \sum_{1 \leq i<j \leq n} t^{-1+i} \tilde{D}_{j} \tilde{D}_{i} \tilde{Y}_{i}^{-1} \\
& =t^{1-n} \sum_{i=1}^{n} D_{i} Y_{i}^{-1} D_{i} .
\end{aligned}
$$

\subsection{Non-symmetric shifted Macdonald polynomials.}

In [1] it was observed that the symmetric ASC polynomials $V_{\lambda}^{(a)}(x)$ coincide (up to a factor and change of variables) with the shifted Macdonald polynomials when $a=0$. We show now that this behaviour carries over to the non-symmetric case.

Following Knop [14], Knop and Sahi [15] and Sahi [26], the non-symmetric shifted Macdonald polynomials $G_{\eta}(z)$ are defined, in the notation of [14], as the unique polynomial with expansion

$$
G_{\eta}(z ; q, t)=\widetilde{E}_{\eta}(z)+\sum_{|\nu|<|\eta|} b_{\eta \nu} \widetilde{E}_{\nu}(z)
$$

which vanishes at the points $z=t^{\bar{\xi}}$ for all compositions $\xi \neq \eta$ such that $|\xi| \leq|\eta|$. Here $t^{\bar{\xi}}$ is given by (1.5). Equivalently $[\mathbf{1 3}, \mathbf{2 2}]$ they can be defined as eigenfunctions of the "inhomogeneous" Cherednik operators

$$
\Xi_{i}=\widetilde{Y}_{i}+\widetilde{D}_{i}
$$

where the operators are defined with the variables $z_{i}$. For such polynomials, Knop [14] defined a raising operator $\Phi_{K}=\left(z_{n}-t^{1-n}\right) \omega^{-1}$ with a simple 
action on $G_{\eta}(z ; q, t)$. It is easily seen that

$$
\lim _{a \rightarrow 0} \frac{-a}{q} \Psi_{1}^{*}=\left.\widetilde{\Phi}_{K}\right|_{z_{i}=t^{n-1} x_{i}}, \quad \lim _{a \rightarrow 0} h_{i}=\left.\widetilde{\Xi}_{i}\right|_{z_{i}=t^{n-1} x_{i}}
$$

which immediately implies the sought relationship between $G_{\eta}$ and $E_{\eta}^{(V)}$.

\section{Proposition 3.11.}

$$
\left.E_{\eta}^{(V)}(x ; q, t)\right|_{a=0}=t^{-(n-1)|\eta|} G_{\eta}\left(t^{n-1} x ; q^{-1}, t^{-1}\right)
$$

or equivalently

$$
\left.E_{\eta}^{(U)}(x ; q, t)\right|_{a=0}=t^{(n-1)|\eta|} G_{\eta}\left(t^{1-n} x ; q, t\right) .
$$

One immediate application of (3.37) is the evaluation of $G_{\eta}(0 ; q, t)$, which follows from (3.27). This is a special case of a result of Sahi [26, Th. 1.1], in which an evaluation formula is given for $G_{\eta}\left(\alpha t^{\bar{\delta}} ; q, t\right)$, for a general scalar $\alpha$. In fact use of (3.37) also allows this more general evaluation formula to be deduced.

Proposition 3.12. With $(\alpha)_{\lambda}^{(q, t)}:=\prod_{s \in \lambda}\left(t^{l^{\prime}(s)}-q^{a^{\prime}(s)} \alpha\right)$ we have

$$
G_{\eta}\left(t^{-\bar{\delta}} \alpha ; q, t\right)=\alpha^{|\eta|}(1 / \alpha)_{\eta^{+}}^{(q, t)} t^{-(n-1)|\eta|} \frac{e_{\eta}}{d_{\eta}} .
$$

Proof. Choosing $a=0$ and $y=t^{n-1-\bar{\delta}} \alpha$ in (3.23), and using (3.30) and (3.38), we see that

$$
\sum_{\eta} \alpha^{-|\eta|} t^{(n-1)|\eta|} \frac{d_{\eta}}{d_{\eta}^{\prime} e_{\eta}} G_{\eta}\left(t^{-\bar{\delta}} \alpha ; q, t\right) E_{\eta}(z)=\prod_{i=1}^{n} \frac{\left(z_{i} / \alpha ; q\right)_{\infty}}{\left(z_{i} ; q\right)_{\infty}} .
$$

But we know that $[\mathbf{1 7}, \mathbf{1 2}]$

$$
\prod_{i=1}^{n} \frac{\left(z_{i} / \alpha ; q\right)_{\infty}}{\left(z_{i} ; q\right)_{\infty}}=\sum_{\lambda} \frac{(1 / \alpha)_{\lambda}^{(q, t)}}{d_{\lambda}^{\prime}} P_{\lambda}(z ; q, t)=\sum_{\eta} \frac{(1 / \alpha)_{\eta^{+}}^{(q, t)}}{d_{\eta}^{\prime}} E_{\eta}(z) .
$$

The result follows by equating coefficients of $E_{\eta}(z)$.

\section{6. $q$-binomial coefficients.}

Sahi [26] uses the polynomials $G_{\eta}$ to introduce non-symmetric $q$-binomial coefficients $\left[\begin{array}{l}\eta \\ \nu\end{array}\right]_{q, t}$ according to

$$
\left[\begin{array}{l}
\eta \\
\nu
\end{array}\right]_{q, t}:=\frac{G_{\nu}\left(t^{\bar{\eta}}\right)}{G_{\nu}\left(t^{\bar{\nu}}\right)}
$$

$\left(\bar{\eta}_{i}\right.$ is defined by (1.5)). Our generating function characterization of the ASC polynomials, and thus by Proposition 3.11 of the polynomials $G_{\eta}$, makes it natural to extend Lassalle's [16] definition of the symmetric $q$-binomial 
coefficients to the non-symmetric case by defining the non-symmetric $q$ binomial coefficients $\left(\begin{array}{l}\eta \\ \nu\end{array}\right)_{q, t}$ according to the generating function formula

$$
\widetilde{E}_{\nu}(x) \prod_{i=1}^{n} \frac{1}{\left(x_{i} ; q\right)_{\infty}}=\sum_{\eta}\left(\begin{array}{l}
\eta \\
\nu
\end{array}\right)_{q, t} t^{l(\eta)-l(\nu)} \frac{d_{\nu}^{\prime}}{d_{\eta}^{\prime}} \widetilde{E}_{\eta}(x) .
$$

We can then use the generating function (3.15) to relate these binomial coefficients to the polynomials $G_{\eta}$.

Proposition 3.13. With $\left(\begin{array}{l}\eta \\ \nu\end{array}\right)_{q, t}$ defined by (3.40), we have

$$
\frac{G_{\eta}(x)}{G_{\eta}(0)}=\sum_{\nu}\left(\begin{array}{l}
\eta \\
\nu
\end{array}\right)_{q^{-1}, t^{-1}} \frac{\widetilde{E}_{\nu}(x)}{G_{\nu}(0)} .
$$

Proof. Multiply both sides of (3.40) by $q^{a(\nu)} t^{(n-1)|\nu|-l^{\prime}(\nu)} \frac{d_{\nu}}{d_{\nu}^{\prime} e_{\nu}} E_{\nu}(y)$ and sum over $\nu$, rewriting the 1.h.s. according to (3.15). Now equate coefficients of $\widetilde{E}_{\nu}(x)$ on both sides. The result then follows upon using (3.28) and (3.37).

Since (3.41) is a formula satisfied by the non-symmetric $q$-binomial coefficients of Sahi [26, Cor. 1.3], and this formula suffices to implicitly define these coefficients, we have that

$$
\left(\begin{array}{l}
\eta \\
\nu
\end{array}\right)_{q, t}=\left[\begin{array}{l}
\eta \\
\nu
\end{array}\right]_{q, t}
$$

Finally, let us present some formulas relating the coefficients $\left(\begin{array}{l}\eta \\ \nu\end{array}\right)_{q, t}$ to their symmetric counterparts $\left(\begin{array}{c}\kappa \\ \mu\end{array}\right)_{q, t}$, which can be characterized by either of the formulas $[\mathbf{1 6}, \mathbf{2 1}]$

$$
\begin{aligned}
P_{\mu}(x ; q, t) \prod_{i=1}^{n} \frac{1}{\left(x_{i} ; q\right)_{\infty}} & =\sum_{\lambda}\left(\begin{array}{l}
\lambda \\
\mu
\end{array}\right)_{q, t} t^{b(\lambda)-b(\mu)} \frac{d_{\mu}^{\prime}}{d_{\lambda}^{\prime}} P_{\lambda}(x ; q, t), \\
\frac{P_{\lambda}^{*}\left(y ; q^{-1}, t^{-1}\right)}{P_{\lambda}^{*}\left(0 ; q^{-1}, t^{-1}\right)} & =\sum_{\mu}\left(\begin{array}{l}
\lambda \\
\mu
\end{array}\right)_{q, t} \frac{P_{\mu}\left(y t^{\bar{\delta}} ; q, t\right)}{P_{\lambda}^{*}\left(0 ; q^{-1}, t^{-1}\right)}
\end{aligned}
$$

Here $P_{\lambda}^{*}$ is the shifted Macdonald polynomial, which is related to the symmetric ASC polynomial $V_{\lambda}^{(0)}$ by [1, Prop. 4.4]

$$
P_{\lambda}^{*}\left(y t^{-\bar{\delta}+n-1} ; q^{-1}, t^{-1}\right)=t^{(n-1)|\lambda|} V_{\lambda}^{(0)}(y ; q, t) .
$$

Proposition 3.14. With $\eta^{+}=\kappa, \nu^{+}=\mu$,

$$
\sum_{\nu: \nu^{+}=\mu}\left(\begin{array}{l}
\eta \\
\nu
\end{array}\right)_{q, t}=\left(\begin{array}{l}
\kappa \\
\mu
\end{array}\right)_{q, t}
$$




$$
\frac{d_{\kappa}^{\prime}}{d_{\mu}^{\prime}} \frac{P_{\kappa}\left(t^{\bar{\delta}}\right)}{P_{\mu}\left(t^{\bar{\delta}}\right)} \frac{d_{\nu}^{\prime}}{E_{\nu}\left(t^{\bar{\delta}}\right)} \sum_{\eta: \eta^{+}=\kappa}\left(\begin{array}{l}
\eta \\
\nu
\end{array}\right)_{q, t} \frac{E_{\eta}\left(t^{\bar{\delta}}\right)}{d_{\eta}^{\prime}}=\left(\begin{array}{c}
\kappa \\
\mu
\end{array}\right)_{q, t} .
$$

Proof. The proof follows the strategy given in [1] for the proof of the corresponding results in the $q=t^{\alpha}, q \rightarrow 1$ limit (binomial coefficients associated with non-symmetric Jack polynomials). For (3.46) we apply the $U^{+}$operator to (3.41), making use of (3.17) and (3.31). Use of the fact that

$$
\frac{a_{\nu}}{E_{\nu}^{(V)}(0)}=\frac{[n]_{t} !}{V_{\eta^{+}}^{(0)}(0 ; q, t)}
$$

and (3.45) then gives

$$
\frac{P_{\lambda}^{*}\left(x t^{-\bar{\delta}} ; q^{-1}, t^{-1}\right)}{P_{\lambda}^{*}\left(0 ; q^{-1}, t^{-1}\right)}=\sum_{\nu}\left(\begin{array}{l}
\eta \\
\nu
\end{array}\right)_{q, t} \frac{P_{\nu^{+}}(x ; q, t)}{P_{\nu^{+}}^{*}\left(0 ; q^{-1}, t^{-1}\right)} .
$$

Comparison with (3.44) implies (3.46) The identity (3.47) follows similarly, by applying $U^{+}$to (3.40) and comparing with (3.43).

\section{Acknowledgements.}

The authors thank the referee for helpful comments. They acknowledge the financial support of the Australian Research Council.

\section{References}

[1] T.H. Baker and P.J. Forrester, Multivariable Al-Salam \& Carlitz polynomials associated with type A q-Dunkl operators, q-alg/9706006, to appear in Math. Nach.

[2] - The Calogero-Sutherland model and polynomials with prescribed symmetry, Nucl. Phys., B 492 (1997), 682-716.

[3] _ A q-analogue of the type A Dunkl operator and integral kernel, Int. Math. Res. Not., 14 (1997), 667-686.

[4] _ Non-symmetric Jack polynomials and integral kernels, Duke. J. Math., 95 (1998), 1-50.

[5] I. Cherednik, A unification of the Knizhnik-Zamolodchikov and Dunkl operators via affine Hecke algebras, Inv. Math., 106 (1991), 411-432.

[6] _ Integration of quantum many-body problems by affine KnizhnikZamolodchikov equations, Commun. Math. Phys., 106 (1994), 65-95.

[7] _ Non-symmetric Macdonald polynomials, Int. Math. Res. Not., 10 (1995), 483-515.

[8] C.F. Dunkl, Differential-difference operators associated to reflection groups, Trans. Amer. Math. Soc., 311 (1989), 167-183.

[9] S. Kakei, Common algebraic structure for the Calogero-Sutherland models, J. Phys., A 29 (1996), 619-624.

[10] _ Intertwining operators for a degenerate double affine Hecke algebra and multivariable orthogonal polynomials, J. Math. Phys., 39 (1998), 4993-5006.

[11] J. Kaneko, Selberg integrals and hypergeometric functions associated with Jack polynomials, SIAM J. Math. Anal., 24 (1993), 1086-1110. 
$[12]$ , q-Selberg integrals and Macdonald polynomials, Ann. Sci. Éc. Norm. Sup., $4^{e}$ série, 29 (1996), 1086-1110.

[13] F. Knop, Integrality of two variable Kostka functions, J. Reine Ang. Math., 482 (1997), 177-189.

[14] _ Symmetric and non-symmetric quantum Capelli polynomials, Commentarii Mathematici Helvetici, 72 (1997), 84-100.

[15] F. Knop and S. Sahi, Difference equations and symmetric polynomials defined by their zeros, Int. Math. Res. Not., 10 (1996), 473-486.

[16] M. Lassalle, Coefficients binomiaux généralisés et polynômes de Macdonald, preprint.

[17] I.G. Macdonald, Hypergeometric functions, Unpublished manuscript.

[18] _ Affine Hecke algebras and orthogonal polynomials, Séminaire Bourbaki, 47ème année, Publ. I. R. M. A. Strasbourg, 797 (1994-95).

[19] Symmetric functions and Hall polynomials. Oxford University Press, Oxford, 2nd edition, 1995.

[20] K. Mimachi and M. Noumi, A reproducing kernel for nonsymmetric Macdonald polynomials, Duke Math. J., 91 (1998), 621-634.

[21] A. Okounkov, Binomial formula for Macdonald polynomials, Math. Res. Lett., 4 (1997), 533-553.

[22] _ Shifted Macdonald polynomials: q-integral representation and combinatorial formula, Comp. Math., 112 (1998), 147-182.

[23] E.M. Opdam, Harmonic analysis for certain representations of graded Hecke algebras, Acta Math., 175 (1995), 75-121.

[24] M. Rösler, Generalized Hermite polynomials and the heat equation for Dunkl operators, Commun. Math. Phys., 192 (1998), 519-541.

[25] S. Sahi, A new scalar product for nonsymmetric Jack polynomials, Int. Math. Res. Not., 20 (1996), 997-1004.

[26] _ The binomial formula for nonsymmetric Macdonald polynomials, Duke J. Math., 94 (1998), 465-477.

[27] K. Takemura, The Yangian symmetry in the spin Calogero model and its applications, J. Phys., A 30 (1997), 6185-6204.

[28] H. Ujino and M. Wadati, Algebraic construction of the eigenstates for the second conserved operator of the quantum Calogero model, J. Phys. Soc. Japan, 65 (1996), 653-656.

[29] _ Orthogonality of the Hi-Jack polynomials associated with the Calogero model, J. Phys. Soc. Japan, 66 (1997), 345-350.

Received December 9, 1997 and revised June 25, 1999.

KYOTO UNIVERSITY

Куото 606-8502

JAPAN

E-mail address: tbaker@kurims.kyoto-u.ac.jp

University of Melbourne

PARKVILLE 3052

Australia

E-mail address: matpj@@ms.unimelb.edu.au 